\title{
REVISTAACIÓN
}

Revista Educación

ISSN: 0379-7082

ISSN: 2215-2644

revedu@gmail.com

Universidad de Costa Rica

Costa Rica

\section{El desempeño del docente como factor asociado a la calidad educativa en América Latina}

\author{
Escribano Hervis, Elmys \\ El desempeño del docente como factor asociado a la calidad educativa en América Latina \\ Revista Educación, vol. 42, núm. 2, 2018 \\ Universidad de Costa Rica, Costa Rica \\ Disponible en: http://www.redalyc.org/articulo.oa? $\mathrm{id}=44055139021$ \\ DOI: https://doi.org/10.15517/revedu.v42i2.27033
}

Esta obra está bajo una Licencia Creative Commons Atribución-NoComercial-SinDerivar 3.0 Internacional. 


\section{El desempeño del docente como factor asociado a la calidad educativa en América Latina}

The Performance of Teachers as a Factor Associated with Educational Quality in Latin America

Elmys Escribano Hervis [1]

DOI: https://doi.org/10.15517/revedu.v42i2.27033

Universidad de Matanzas, Cuba

escriba2003@gmail.com

Redalyc: http://www.redalyc.org/articulo.oa?id=44055139021

Recepción: 24 Noviembre 2016

Aprobación: 30 Mayo 2018

\section{ReSUMEN:}

El tema de este ensayo se ubica en los estudios actuales sobre calidad de la educación y, en este caso, la calidad asociada al desempeño docente, esta relación aparece contextualizada en América Latina. El propósito que se sigue con este trabajo es argumentar la significación y el lugar que ocupa el desempeño docente como factor directamente asociado a la calidad educativa. Se sostienen algunas ideas básicas: a saber, América Latina no ha satisfecho metas elementales en cuanto a equidad y justicia en el acceso a la educación, lo que representa un lastre en el trabajo por la calidad de este servicio; la calidad educativa está asociada a un conjunto de factores entre los que se cuentan los factores económicos, políticos, culturales, científicos y tecnológicos, así como un importante factor humano: el desempeño docente, que resulta vital según la postura que se sostiene. En ese orden, se señalan las limitaciones que se presentan hoy en el proceso de formación inicial y continua del personal docente, así como los desafíos que debe encarar como parte de su desempeño para brindar una respuesta coherente que implica una educación de calidad en el presente siglo y los cambios sociales, culturales y tecnológicos que se viven a diario. Este ensayo es la expresión de una sistematización teórica, sobre la base de consultas de diversas fuentes sobre el tema, en la que se resaltan, por su valor, informes de organismos internacionales.

Palabras ClaVe: Educación, formación del profesorado, desempeño docente, calidad de la educación.

\section{ABSTRACT:}

The theme of this essay is located in the current studies on the quality of education and, in this case, the quality associated with the teacher's performance, this relationship appears contextualized in Latin America. The purpose of this work is to argue the significance and place of the teacher's performance as a factor directly associated with educational quality. Some basic ideas are supported: namely, Latin America has not met basic goals in terms of equity and justice in access to education, which represents a burden on the work for the quality of this service; Educational quality is associated with a set of factors including economic, political, cultural, scientific and technological factors, as well as an important human factor: the teacher's performance, which is vital according to the position held. In that order, the limitations that are presented today in the initial and continuing teacher training process are pointed out, as well as the challenges that must be faced as part of their performance to provide a coherent response that implies a quality education in the present century and the social, cultural and technological changes that are experienced daily. This essay is the expression of a theoretical systematization, based on inquiries from various sources on the subject, in which reports from international organizations stand out for their value.

KEYWORDS: Education, teacher training, teaching performance, quality of education.

\section{NotAS DE AUTOR}

[1] Licenciado en Pedagogía - Psicología (Instituto Superior Pedagógico “Enrique José Varona”, La Habana, 1985) Doctor en Ciencias Pedagógicas (Instituto Central de Ciencias Pedagógicas, La Habana, 1998) y Profesor Titular de la Universidad Matanzas. Fungió desde mayo 1997, hasta julio de 2014 como Vice-Rector de Investigaciones, Postgrados y Relaciones Internacionales de la Universidad de Ciencias Pedagógicas de Matanzas. Su tesis de grado: "La concepción de la educación en la obra de José Martí", fue merecedora del Premio Nacional a la mejor Tesis de Grado, fue publicada en el año 2006, por el editorial Pueblo y Educación, esta misma editorial publicó en el año 2011 otro texto de su autoría "José Martí para educadores. Biografía", ha publicado el resultado de sus investigaciones en revistas cubanas y extranjeras. Es además Coordinador del Simposio Internacional Educación y Cultura en Iberoamérica, Presidente del Comité Académico del programa -certificado- de la Maestría en Educación de su Universidad. Es Director de la revista ATENAS y Miembro de la Junta de Acreditación Nacional. Es miembro del Tribunal Estatal de Grado Científico de Ciencias de la Educación y miembro del Consejo Técnico Asesor del Ministerio de Educación. Es experto del Programa Nacional: Problemas actuales del Sistema Educativo Cubano. Perspectivas de desarrollo. Ha integrado claustros de formación doctoral en universidades de México y Venezuela. Ha fungido como consultor internacional en el Ministerio de Educación de Nicaragua. 


\section{INTRODUCCIÓN}

La reflexión sobre la educación con una base crítica en la actualidad, resulta vital para las ciencias y para la sociedad. Los resultados de las investigaciones - histórico y comparativas- y de las evaluaciones a nivel nacional e internacional deben servir de base para pronosticar la dinámica de los cambios y tomar las decisiones requeridas de forma oportuna.

Para todas las áreas del mundo, pero en especial para América Latina, los estudios y la reflexión actual sobre el tema debe tener siempre en cuenta la historia, pues para el caso particular de la educación en su devenir histórico, en nuestros países, acumula una serie de insatisfacciones y carencias que es necesario observar para comprender la situación actual y estar en condiciones de favorecer la proyección del desarrollo futuro (Escribano, 2017). Los estudios comparados de la educación, también aportan elementos de interés, que ninguna nación o Estado debe obviar.

Realizar una valoración integral ajustada a la problemática social - educativa y que capte la esencia de todo el continente resulta complejo y riesgoso, en tanto América Latina, hoy se identifica por una gran diversidad de culturas y niveles de desarrollo, entre sus países e, incluso, existe diversidad entre cada región al interior de estos.

Para el caso de la reflexión que se presenta en esta ocasión, su propósito básico es valorar algunas problemáticas actuales relacionadas con la calidad de la educación correlacionada con el desempeño del personal docente ante las demandas y desafíos de la sociedad y la cultura del siglo XXI en diferentes contextos del continente.

Las ideas que se exponen toman en cuenta un conjunto de posturas y puntos de vista -ofrecidos por relevantes investigaciones y analistas del tema, así como informes emitidos por organizaciones internacionales- que resultan hoy referentes esenciales sobre la reflexión actual referida a la educación, enjuiciada de manera crítica por sus debilidades y carencia de logros sostenidos.

El ensayo plantea una lógica expositiva que parte de considerar las insatisfacciones y críticas más comunes que hoy se le plantea a la educación y a la escuela. Posteriormente, se señalan algunos aspectos que deben ser contemplados al evaluar una educación de calidad, pues son factores estrechamente vinculados a esta condición y, finalmente, se enjuicia el proceso de formación del personal docente en América Latina, el cual es considerado un factor relevante en términos de calidad educativa. En el último apartado del ensayo se sitúan cinco áreas que se consideran clave en el desempeño docente para una educación de calidad en el contexto de las demandas del siglo XXI.

\section{INSATISFACCIONES SOBRE LA CALIDAD DE LA EDUCACIÓN}

En el ámbito de los estudios actuales sobre la educación resalta la insatisfacción y la crítica a los sistemas educativos por la débil pertinencia expresada en la inoperancia de la formación que se dispensa en las instituciones educativas y las necesidades que señala la vida social, el desarrollo de la ciencia, la tecnología y el propio ser humano en sus actividades vitales.

El análisis actual de la educación, la escuela, el pensamiento educativo, las tendencias al uso, así como el complejo tema de la calidad de la educación y los factores influyentes contextualizado en América Latina, debe tomar en cuenta, de forma crítica, las condiciones que imponen las prácticas neoliberales y la imposición de su racionalidad instrumental (Burchardt, 2017; Guadarrama, 2008).

La influencia neoliberal hace ver la educación desde una perspectiva "economicista", en la que importa su rentabilidad, además, se hacen comunes criterios de eficientismo, así como el desarrollo rápido de determinadas competencias en detrimento de la necesaria formación humanística que debe identificar a cualquier docente (Guadarrama, 2008; Torres, 2001). 
Las asimetrías de los sistemas educativos destacan en varios sentidos y dimensiones de análisis en la integralidad y la coherencia necesaria de estos sistemas, su actualidad en conformidad con su tiempo y su cultura, así como la polémica insatisfacción manifiesta por parte de docentes ante las reformas que históricamente se han llevado a cabo. Estos elementos inciden, de modo directo, en la calidad del ofrecimiento educativo que dispensa la sociedad.

Entre las insatisfacciones que se le señala a la educación y a la escuela contemporánea, especialmente en América Latina, están las siguientes:

- La existencia de millones de personas con analfabetismo absoluto recuerda a diario la incapacidad de los sistemas educativos del área y sus correspondientes sociedades de solucionar de manera definitiva una problemática de la época colonial, instalada hoy en pleno siglo XXI; a esto se le adicionan otros tipos de analfabetismo contemporáneo: el funcional y el digital, que complejiza de manera notable, logros sostenibles en el trabajo por la calidad (Martínez, Truco \& Palma, 2014; SITEAL, 2013)

- No se garantiza el acceso pleno con igualdad de posibilidades para la inclusión social de todas las personas en el sistema educativo. La escuela es incapaz de tratar a cada quien en correspondencia con sus diferencias de origen (etnias, culturas, religión, procedencia social, género, etc.). En este punto y en el anterior se sostiene la debilidad de las políticas educativas como una de las causantes fundamentales.

- No se enseña a pensar y obtener información por sí, de forma crítica, para propiciar aprendizajes autónomos para toda la vida.

- No se estimula el diseño propio de proyectos de vida que permitan conducirse con satisfacción y autonomía.

- No se desarrolla la creatividad, la innovación y el espíritu crítico desde la escuela.

- No se brinda respuesta a las necesidades de formación de una sociedad de la información que tiende al uso creciente de las TIC en la vida social y la cultura (Adams Becker \& et al, 2017; SITEAL, 2014; Sunkel, Trucco \& Espejo, 2013; UNESCO, 2013).

\section{LA CALIDAD DE LA EDUCACIÓN Y LOS FACTORES ASOCIADOS, SU VÍNCULO CON EL DESEMPEÑO DOCENTE. EL PUNTO DE PARTIDA}

Es justo que nuestros países piensen y trabajen, prioritariamente, por una educación de calidad para todos los sectores o grupos, con una escuela inclusiva que sea expresión de justicia social, como una meta alcanzable. Este proyecto no puede ser infinitamente postergado para generaciones futuras. No están reñidas la masividad y la calidad. Hoy en nuestro continente la desigualdad y la pobreza son los principales obstáculos y amenazas para alcanzar esta meta. La situación de pobreza limita el acceso a la educación y obstaculiza el aprovechamiento del estudio y de las oportunidades educativas por parte de los alumnos y alumnas. Es necesario apreciar, de modo dinámico y multidimensional, el problema de la pobreza y su incidencia en la educación en América Latina, en relación con los diferentes indicadores que pueden ser considerados y con la combinación en que se manifiesten en cada contexto en la vida social (García, Caamal, \& Prieto, 2016; García, 2014; Pérez, 2003; Plá, 2014; Tedesco, 2010). Desde el punto de vista metodológico, la influencia de la pobreza y la exclusión es un condicionamiento de primer orden a valorar, en los estudios acerca de la educación y su calidad como parte de la variable: contexto.

La calidad de la educación es una problemática compleja como proceso y como resultado. También resulta compleja su definición, un concepto multidimensional, en el cual el logro de aprendizajes es un elemento imprescindible, entre otros aspectos de interés que le dan sentido e integralidad a la percepción de este objeto (Van de Velde, 2016). Este autor, señala: 
Calidad educativa es necesariamente calidad para todas y todos. Al no ser así, entonces NO es calidad. Implica educación incluyente, sin discriminación, con respeto a la diversidad, incluso su disfrute sincero. Integra y se basa en cooperación genuina, como eje educativo integrador e incluyente por excelencia (p. 2).

En opinión de (Román \& Murillo, 2008): "Mientras más pobres y excluidos, más pobre e insuficiente es la educación a la que acceden" (p. 1).

La (OREALC/UNESCO, 2007) señala que la educación de calidad es un derecho fundamental de todos los individuos, cuyas características principales son el respeto a los derechos, la equidad, la relevancia y la pertinencia, además de dos componentes de carácter operativo: la eficacia y la eficiencia (Martínez-Chairez, Guevara-Araiza, \& Valles-Ornelas, 2016).

De acuerdo con la UNESCO (2007), la educación de calidad se refiere a variables como eficiencia (buen uso de recursos), equidad (distribución de beneficios educativos), relevancia (que responda a las necesidades de la sociedad) y pertinencia (que responda a las necesidades del estudiantado) (OREALC/UNESCO, 2016).

Como se aprecia, la calidad de la educación depende de la cantidad de recursos, la infraestructura y el soporte material, pero sobre todo, del empleo que se hace de estos; de cómo se organiza y gobierna el sistema educativo; de cómo se forma al profesorado; y, de la motivación, el apoyo y la participación de los grupos sociales o agentes educativos implicados, al mismo tiempo que subraya la significación de las adquisiciones y logros del aprendizaje para la vida en cada estudiante en cada nivel educativo. En este orden, la relevancia y la pertinencia de la educación resultan ideas clave para orientar el análisis en función de la calidad. Un elemento básico que identifica la educación de calidad es la distribución equitativa de oportunidades con las garantías requeridas de acceso para que cada estudiante concluya de manera exitosa un grado, un ciclo o un nivel educativo. La UNESCO señala: "La calidad de la educación requiere de un entorno inclusivo y democrático, donde todos los niños puedan desarrollar al máximo sus potencialidades” (OREALC/UNESCO, 2012, p. 79).

Se sostiene que no es posible hablar de calidad de educación cuando pesa sobre el sistema educativo, exclusión, falta de acceso, analfabetismo -sin importar su naturaleza-, inequidad, abandono o deserción escolar, unido a otros elementos como son la irrelevancia de los contenidos, su falta de vínculo con la vida o desactualización y un pobre desempeño del personal docente.

La educación en cualquiera de sus niveles de realización y logros está asociada a factores económicos, políticos, tecnológicos, culturales y humanos. Su proyección y alcance está directamente relacionado con un ideal de sociedad. "Una educación de calidad para todos no es un proyecto educativo, es un proyecto de sociedad" (Tedesco, 2010).

El desempeño docente es un factor que se asocia de manera directa a la calidad de la educación (MartínezChairez, Guevara-Araiza, \& Valles-Ornelas, 2016). La sociedad y la escuela deben contar con profesionales con buena preparación y con clara conciencia del alcance de su desempeño. Señalan (Román \& Murillo, 2008): "los docentes son actores claves y relevantes para la calidad de los procesos de enseñanza y aprendizaje es algo de lo cual hay no sólo consenso social, sino que cada vez es más sólida la evidencia empírica que lo confirma” (p. 2).

Resulta conclusiva y básica como principio para la acción, la afirmación de Juan Carlos Tedesco (2010): "la calidad de la educación la definen los maestros y los profesores, ningún país ofrece mejor educación o educación de mayor calidad más allá de la calidad de sus maestros”.

La educación es un tipo especial de relación humana, por tanto, en este escenario se requieren componentes de preparación, motivación y conciencia que identifiquen el desempeño profesional a la altura de las exigencias de la época y la sociedad en que se enmarca. "El pilar fundamental de la calidad educativa son las capacidades profesionales docentes: si los alumnos no se encuentran en sus aulas con docentes capaces de generar mayores oportunidades de aprendizaje, el genuino mejoramiento de la calidad educativa no se 
producirá" (Oficina Regional de Educación para América Latina y el Caribe [OREALC/UNESCO], 2013, p. 11).

Otro concepto que resulta básico para el presente artículo en la temática objeto de análisis, como ya se ha visto, es la correspondiente toma de posición acerca del desempeño docente, o desempeño profesional pedagógico, o desempeño profesional del educador o educadora, etc. Llama la atención, en un primer nivel de análisis, las variadas denominaciones que hacen alusión o designan el mismo objeto (Medina, 2017).

El desempeño del personal docente integra dentro de sus significados la capacidad, la competencia, el proceso, o el modo de actuación, entre otras.

(Pérez R. J., 2008) lo define del siguiente modo:

El conjunto de acciones que con alta motivación, preparación pedagógica y creatividad realiza el profesor, durante el desarrollo de su actividad, lo cual se manifiesta, tanto en el proceso como en el resultado de esta, orientada a lograr la formación integral de la personalidad de cada uno de sus alumnos, con un enfoque desarrollador y diferenciado, tomando como base el conocimiento de sus posibilidades y, en particular, sus necesidades, motivaciones, vivencias y proyectos de vida. (p. 66)

Por su parte, (Pérez \& et al, 2009), definen este desempeño profesional como:

El conjunto de acciones que realiza el maestro, durante el desarrollo de la actividad pedagógica, que se concretan en el proceso de cumplimiento de sus funciones básicas y en sus resultados, para lograr el fin y los objetivos formativos del nivel educativo en el que trabaje. (p. 21)

Existe coincidencia (Medina, 2017; Pérez, 2009; Pérez, 2008) al considerar que el desempeño de docente se manifiesta a través de las acciones que desarrolla el profesorado en su actividad profesional, la cual responde a los objetivos propios de la actividad pedagógica, para favorecer el cumplimiento de las funciones relacionadas con su labor, la cual exige la presencia de un conjunto de cualidades personales y condiciones que se erigen como condicionantes necesarias para la adecuada materialización de estas acciones.

El desempeño del personal docente como concepto, hace referencia e integra la lógica que le brinda sentido a su labor profesional:

- El dominio profundo - con relevancia y pertinencia- del sistema de contenidos actualizados sobre la disciplina que debe impartir.

- La necesaria y actualizada preparación que debe poseer, sustentada en profundos conocimientos en el campo de las ciencias de la educación, especialmente de la pedagogía, la psicología, la didáctica, así como la metodología de la investigación educativa.

- Desarrollo adecuado de competencias profesionales en el ámbito de la comunicación, el empleo sistemático de las TIC y otros aspectos de relevancia.

- La necesidad de un componente ético, con altas motivaciones profesionales y personales en el ejercicio de su labor con calidad, en una gestión de esta misma de manera constante, tanto en los procesos como en los resultados.

- El imprescindible conocimiento de las condiciones particulares de cada contexto -escolar - familiar - comunitario- donde desarrolla sus acciones profesionales y la necesaria claridad y precisión del fin esencial de su labor profesional.

La preparación para el desempeño del personal docente comienza desde la etapa de los estudios de pregrado, ya que este momento ofrece condiciones favorables para su preparación con vista a su futura labor profesional. Al respecto, (Parra, 2000) apunta que "la etapa de pregrado marca pautas decisivas para formar una actitud ante la profesión y desarrollar las bases del futuro desempeño” (p. 32). 


\section{El PROCESO DE FORMACIÓN INICIAL DEL PERSONAL DOCENTE EN AMÉRICA LATINA}

El desempeño docente como elemento humano y relacional en la educación resulta esencial. Es preciso que la formación inicial del profesorado responda, de forma directa, a las necesidades del contexto en correspondencia con una filosofía de la educación que esté vinculada a la historia y a la cultura (Chávez, 1995). Esta filosofía debe fecundar cada elemento que se integra al proceso de formación y perfeccionamiento continuo del personal docente.

Para la concepción adecuada de su formación inicial, se necesita prioritariamente, diseñar un modelo educativo que oriente, de forma coherente, como filosofía de la educación, todo el sistema educativo en todas sus partes integrantes y relaciones al interior de dicho sistema y que se conecte con el "sistema nervioso" de la sociedad y el referente que significa la cultura en la cual se contextualiza.

Para tener un nuevo modelo educativo en Latinoamérica que aborde la diversidad en la unidad, se requieren enfoques o propuestas pedagógicas contextualizadas a los procesos socio-económicos y culturales de la región, como en su momento lo fue la pedagogía de Paulo Freire y el movimiento de la escuela nueva, con orientación a la generación de sociedades del conocimiento (Tobón, González, Nambo, \& Vázquez, 2015, p. 6).

El principal referente para el diseño de un sistema educativo debe ser la propia cultura, la historia y lo mejor de la tradición pedagógica de la nación de que se trate. Al mismo tiempo, debe situarse con un sentido crítico en la actualidad, con una determinación clara de las fortalezas y debilidades de dicho sistema en todas sus relaciones y elementos constituyentes y, de ese modo, determinar, en correspondencia con los adelantos de la ciencia y la tecnología, el ideal de calidad que prevalecerá y trazará el camino para toda la sociedad y no solo para la escuela y el desempeño de sus docentes.

Estudios sobre el tema en América Latina (Oficina Regional de Educación para América Latina y el Caribe (OREALC/UNESCO), 2013); (Vaillant, 2007); apuntan diferentes problemáticas que es urgente superar:

- Bajo nivel cognitivo y de desarrollo de habilidades de estudiantes que ingresan a la carrera magisterial. Eso les acarrea dificultades en este tipo de estudios (OREALC/UNESCO, 2013).

- En el proceso formativo, las carreras de formación magisterial no se destacan por brindar un modo de actuación donde se integre creadoramente la investigación y la innovación, así como el uso de novedosos recursos pedagógicos. Más bien, se identifican "por un bajo prestigio, demasiado énfasis en el método basado en la exposición oral frontal y muy poca atención a técnicas pedagógicas apropiadas para los estudiantes socialmente desfavorecidos, clases multigrado, clases multiculturales, aprendizaje de la lecto-escritura y el cálculo, resolución de conflictos" (Vaillant, 2007, p. 3).

Por otra parte, se manifiestan dudas sobre la calidad de los programas o currículos formadores. Un estudio sobre la realidad brasileña, apunta:

El contenido de los programas es considerado fragmentario y disperso en los programas de preparación para la educación primaria con poco énfasis en lo requerido para enseñar en las aulas especialmente en las áreas de contenido disciplinario. Se destaca la dificultad de concretar la articulación entre formación teórica y práctica y el escaso énfasis en aspectos metodológicos de la enseñanza (Gatti \& de Sá Barreto, 2009; Gatti, de Sá Barreto \& André, 2011), citado por: (OREALC/ UNESCO, 2013, p. 41).

Algunos autores, autoras y fuentes señalan como una limitante la diversidad en los tipos de centros formadores (OREALC/UNESCO, 2013). Sin embargo, es importante señalar que, en el caso de América Latina, las escuelas normales que, incluso suelen recibir la mayor parte de las críticas, han desempeñado un rol histórico destacado en la formación de profesionales para los niveles iniciales de educación. Su labor se entreteje en lo más íntimo del espíritu nacional de la identidad y la cultura en nuestros países, estudiantes que han graduado se han identificado por décadas por su profunda preparación profesional y su sólida eticidad. 
- El estatus de la profesión es considerado bajo, lo que no atrae al mejor estudiantado que opta por otras carreras (OREALC/UNESCO, 2013).

En contraste con lo que sucede con estudiantes de carreras distintas a la docencia, el alumnado candidato a ser docentes (maestros y maestras y profesorado, tienen antecedentes socioeconómicos más limitados tanto en los niveles de instrucción de sus padres y madres como en sus niveles de ingreso familiar. "También los estándares de admisión a las instituciones de formación de docentes tienden a ser más bajos que en el conjunto de carreras de educación superior” (Vaillant, 2007, pág. 4).

- El necesario vínculo entre la teoría y la práctica en la formación docente atraviesa por la falta de sistematicidad y de sinergia (Vaillant, 2007).

- El ofrecimiento de la superación y la educación de postgrado, por lo general, no se ha integrado como respuesta a necesidades reales de mejoramiento y desarrollo del desempeño docente, más bien "se ha desarrollado sin apego a reglas de calidad y pertinencia" (OREALC/UNESCO, 2013, p. 74). Esta misma fuente señala:

La limitada inserción de los programas de desarrollo profesional en políticas de largo plazo dirigidas a los docentes. Es decir, en ausencia de un programa coherente y bien coordinado, las actividades propiamente de desarrollo profesional conviven con múltiples iniciativas promovidas desde diversas instancias administrativas y de gestión con la consecuente dispersión y falta de pertinencia para los objetivos relacionados con la profesionalización (OREALC/UNESCO, 2013, p. 70).

- Las condiciones de trabajo resultan desestimulantes, es bajo el reconocimiento social, así como se carece de incentivos a la innovación educativa y a las mejores prácticas y resultados.

Muchos países se caracterizan por un entorno profesional que presenta dificultades a la hora de retener al personal docente idóneo. Son escasos los estímulos para que la profesión docente sea la primera opción de carrera. Las condiciones de trabajo son a menudo inadecuadas y existen serios problemas en la estructura de remuneración y distribución de incentivos. A esto se agrega la falta de una formación inicial y un desarrollo profesional adecuados que les preparen para la tarea de enseñar (Vaillant, 2007).

Otra fuente insiste en la problemática:

Aunque la información disponible es parcial, todo indica que la situación predominante de la docencia en la región no se ajusta a las características de una profesión de alto estatus: los salarios y las condiciones laborales son deficientes, la formación inicial es de baja calidad, y las oportunidades de desarrollo profesional son limitadas. En consecuencia, el desafío de las políticas docentes es monumental: configurar una carrera profesional docente capaz de atraer a jóvenes talentosos a la docencia, formar adecuadamente a los candidatos, retener en las aulas (especialmente en aquellos sectores más desaventajados) a los profesores competentes, y hacer del desarrollo profesional una necesidad y una exigencia. Dado el carácter sistémico de todos estos procesos, es difícil avanzar en uno sin hacerlo en los demás (Oficina Regional de Educación para América Latina y el Caribe (OREALC/UNESCO), 2013, p. 11).

Lo antes apuntado se hace más agudo y complejo de solucionar de manera satisfactoria en las áreas marginales pobres, rurales e indígenas, lo que refuerza la idea de que resulta prioritario para la sociedad diseñar un sistema educativo inclusivo y con justicia social (OEI, 2014). En este caso, la formación inicial del personal docente para trabajar en estos contextos requiere una acentuada preparación pedagógica, cultural y ética con altos niveles de responsabilidad social.

Como se aprecia, la situación que debe transformarse es compleja. Se requiere de la interacción coherente, sistemática y efectiva de un conjunto de factores políticos, económicos, científicos, culturales, tecnológicos y humanos. Aunque la reflexión sobre la variable económica no es el centro de atención de este trabajo, es importante dejar sentado que la inversión en educación en América Latina, hoy es insuficiente si se quiere elevar los estándares en todos los niveles educativos.

Los países de la región invierten cerca del $12 \%$ de sus ingresos per cápita en educación, comparados con cerca del $19 \%$ de los países miembros de la OCDE. Un aumento del orden del $50 \%$ en el gasto per cápita, pondría a los países de la región a la par con los países de más altos niveles de inversión del mundo. Sin 
embargo, dado los ingresos sustancialmente más bajo de nuestros países, esto todavía no sería suficiente. Incluso en el mejor escenario posible, los países tendrán que elegir entre modestos mejoramientos de la educación primaria y secundaria y la expansión de la educación superior (Schwartzman, 2001).

Al valorar la inversión por alumno, que está altamente correlacionada con el peso del PBI. En América Latina el promedio de inversión anual por alumno del nivel secundario rondaba los 2.000 dólares mientras que en los países de Europa Occidental casi alcanzaba los 12.000 dólares con paridad cambiaria. Es indudable que esa diferencia de inversión por alumno tiene efectos diferenciales en la calidad educativa. (Rivas, 2015, p. 194)

Mejorar la calidad de la educación resulta una meta muy difícil e igual de costosa, pues exige educadores y educadoras con mejor formación y remuneración, mejores edificaciones, menos estudiantes por docente, mejor mobiliario y equipamiento en las escuelas, laboratorios bien dotados para todas las disciplinas docentes, bibliotecas actualizadas y computadoras con acceso pleno a internet, repositorios y bases de datos que sean de interés para cada institución educativa (Schwartzman, 2001).

\section{¿QUÉ ELEMENTOS DEBEN REGIR EL CAMBIO EN EL DESEMPEÑO DEL PERSONAL DOCENTE?: UNA PROPUESTA PARA LA ACCIÓN}

En el siguiente apartado, el autor presenta una propuesta de cinco aspectos esenciales en los que se debe centrar el cambio para la formación inicial y permanente de docentes con referencia particular a América Latina. Se han tomado en cuenta los informes de organismos internacionales, así como resultados de investigaciones en torno al tema.

La formación inicial y permanente de docentes, como elemento que se correlaciona con su desempeño profesional y, por ende, con la calidad de la educación, depende en primer lugar de un cambio de la filosofía de la educación que orienta este proceso a nivel social.

Las bases para una filosofía de la educación que derive en una propuesta integradora de acciones debe erigirse en arreglo dialéctico con la sociedad, la historia, el contexto y la cultura. En tal sentido, es necesario cierta dosis de proyección y adelanto, para considerar qué tipo de sociedad y de ser humano necesitamos dentro de 20 o 30 años.

Este elemento que se menciona tiene un carácter básico o más bien de premisa sobre la cual se insertarán iniciativas o acciones que emanan del diálogo enriquecedor con otras culturas y realidades.

Un elemento estimulante para el cambio lo constituye el hecho de que, desde fines del siglo XX, la UNESCO ha estimulado la realización de informes y estudios inspiradores de la educación que debemos dispensar en cada institución, nivel educativo o país (Delors, s. f.; Morin, 2000). Hace poco más de una década se viene promoviendo la reflexión sobre las competencias o habilidades básicas para la educación del siglo XXI. En el año 2013, la Fundación Telefónica tuvo la iniciativa de generar un debate entre analistas sobre cómo debería ser la educación del siglo XXI. Distinto personal experto ha brindado su visión (Albury, 2013; Gerver, 2013; Patti, Brackett, Ferrándiz \& Ferrando, 2011; Savater, 1997; Siemens, 2012). Al mismo tiempo, algunas organizaciones, incluyendo la iniciativa privada, también han generado la reflexión sobre el tema, por ejemplo: la definición y selección de competencias clave (DeSeCo) (OCDE, 2000); habilidades y competencias del siglo XXI para aprendices del milenio en los países de la OCDE (OCDE, 2010); competencias del siglo XXI. Guía práctica para promover su aprendizaje y evaluación (Fundación Omar Dengo, 2014); P21. Framework for 21st Century Learning (P21 Framewok for 21st Century Learning, 2015).

Cada documento generado ofrece una perspectiva particular en correspondencia con la toma de una posición teórica y define las adquisiciones que considera básicas, como habilidad o competencia que resultaría vital en el contexto del siglo XXI, en relación con la dinámica de la vida, la ciencia, la tecnología y la cultura contemporánea. 
La necesaria reformulación de la educación, la determinación de sus bases teóricas y sus presupuestos organizativos deben hacerse explícitos en espacios sociales que abarquen a la familia y demás instituciones sociales; y muy especialmente, a docentes.

Estas determinaciones incluyen el diseño de los currículos para el proceso de formación inicial de docentes en escuelas normales, institutos especializados o universidades; y, en segundo orden, es necesario diseñar, de forma coherente, la formación permanente en el puesto de trabajo con una amplia gama de opciones de superación y educación de postgrado que responda a sus necesidades para el mejoramiento del desempeño profesional. Solo sobre esta base tiene sentido emitir una propuesta de aspectos que se consideran básicos para influir en la calidad de la educación tomando en cuenta la variable clave: el desempeño docente.

Es evidente que las políticas educativas están condicionadas por la historia, los contextos económicos, políticos y culturales, de lo cual ya se ha denotado su diversidad en la región. No obstante, cada país debe determinar la filosofía que rija la educación en correspondencia con sus condiciones y necesidades de formación. Aunque en este tema no existe lugar para fórmulas o recetas, es posible identificar un conjunto de direcciones básicas para la educación; y, como es lógico, los cuerpos docentes deben ser exponentes del desarrollo de estas como parte de su desempeño. Estas direcciones deben ser desarrolladas en la institución educativa con niveles crecientes de integración y complejidad con el resto de los agentes educativos que influyen en los procesos de formación humana.

- Aprender a aprender y hacerlo a lo largo de toda la vida

Resulta una respuesta urgente y necesaria a la lógica del desarrollo de la sociedad y la cultura que genera crecientes volúmenes de información. La escuela debe dejar de ser el lugar que transmita saberes acabados, para ser el lugar que desarrolle la competencia de aprender a aprender activando en cada ser humano recursos personológicos propios para satisfacer esta necesidad a lo largo de su existencia. Es preciso desarrollar las habilidades de búsqueda, selección, clasificación y procesamiento de la información disponible en diferentes formatos. Se señala que ya para el período entre 1955 y 1970, solo 15 años, eran necesarios para duplicar la información disponible en el mundo. En 1988 y 1993, eran necesarios 5 años. Las estimaciones señalan, que para el 2020, serán solo necesarios 75 días para duplicar todo el volumen de información disponible en el mundo. Esto es razón suficiente para que la educación esté enfocada a enseñar a que las personas aprendan por sí mismas.

- Creatividad e innovación

Alude a la necesidad de que la escuela estimule la búsqueda de solución a nuevos problemas o a que sus estudiantes sean capaces de encontrar mejores formas de resolver problemas conocidos. Esta competencia implica la activación de una mezcla de recursos propios de procesos cognitivos y afectivos en estrecho vínculo. Se basa en el empleo o aplicación sistemática del repertorio cultural personal en el que se evidencia la utilidad de lo aprendido para desenvolverse adecuadamente en la vida. Ser una persona creativa e innovadora es un imperativo de la vida contemporánea para enfrentar un futuro que no hemos sido capaces de anticipar; (Van de Velde, 2016) (Gerver, 2013; Robinson, 2011; Van de Velde, 2016). Por tanto, se requiere que la escuela fortalezca la capacidad de observar, la imaginación, se estimule el ofrecimiento de respuestas a problemas haciendo uso de lo que se ha adquirido en vínculo directo con la aplicación a la vida. Es necesario primero comprender con claridad y precisión, para ser capaces de aplicar de modo acertado.

- Pensamiento crítico

El pensamiento crítico se interconecta, de forma directa, con la necesidad de aprender a aprender, en este caso, se refiere a cómo se maneja e incorpora al repertorio personal cultural de cada quien todo el nuevo contenido aprendido. Implica realizar juicios de valor basados en hechos y análisis contrastados con las fuentes consultadas. En tal sentido, en la escuela debe estimularse la consulta de variedad de información en la que se sostengan diferentes argumentaciones y puntos de vista, en los cuales sea necesario comprender y analizar para tomar partido y poder defender juicios personales 
con responsabilidad, y que esto sea válido para la posterior toma de decisiones. El pensamiento crítico debe ser estimulado con la organización del trabajo y las tareas docentes de forma individual y apelando a modos colaborativos (Van de Velde, 2016).

- Colaboración

La escuela debe estimular la convivencia, la socialización y la cooperación. El talento individual debe emplearse de manera compleja en la solución de problemas que impliquen la asunción de posiciones colectivas o grupales. La interdisciplinariedad en las investigaciones, las opciones de comunicación instantáneas con las que se cuentan, reclaman la participación colectiva para solucionar problemas. La colaboración como método de trabajo que debe fomentar la escuela prepara a sus estudiantes para vivir en la sociedad del siglo XXI, presupone la concepción y realización de proyectos que desbordan los marcos de la escuela, hasta llegar a implicar la participación de otro estudiantado y profesorado (Van de Velde, 2014, 2016) El ser humano encuentra su verdadera y genuina expresión individual mediante las relaciones sociales, esto debe ser fomentado desde la escuela con la práctica de un aprendizaje activo y cooperativo.

- Educación mediada por TIC

Las TIC representan una potencialidad significativa para cambiar los modos en que se enseña, se aprende y se colabora. El aprendizaje dejó de ser patrimonio solo del espacio físico que demarca la institución educativa. Hoy el aprendizaje debe ser concebido, como ya se dijo, para toda la vida, pero se puede desarrollar en todos los espacios, de manera individual y colaborativa.

La formación de competencias digitales es cada vez más importante en el ámbito educativo como una necesidad para la inclusión en la sociedad del conocimiento: las TIC no son solo un potente recurso para el aprendizaje, son herramientas cada vez más relevantes para la vida. El potencial de las TIC no se refiere solo a la alfabetización digital, ellas pueden ser utilizadas para promover competencias modernas y mejorar el desempeño educativo del estudiantado en términos generales. En los países de la región [el autor se refiere a Latinoamérica], el acceso a estas nuevas tecnologías en el hogar está fuertemente condicionado por el nivel socioeconómico de las familias, por lo que el sistema escolar ha sido la principal herramienta para reducir esta brecha tecnológica; aunque -ciertamente- esta continúa siendo muy relevante en la mayoría de los países. Además de continuar equipando con TIC a las escuelas donde se educan quienes no pertenecen a los sectores privilegiados, el desafío futuro es cómo lograr que -más allá del uso recreativo- el estudiantado latinoamericano les de un uso con potencial educativo; esto supone capacitar mejor a docentes para que incorporen, a sus prácticas de enseñanza, estas nuevas tecnologías (Oficina Regional de Educación para América Latina y el Caribe (OREALC/UNESCO), 2013).

Como se aprecia, el potencial de las TIC no es mágico; requiere del desempeño docente, con altos y crecientes niveles de responsabilidad, profesionalidad, preparación y capacidad para diseñar un proceso de adquisición para toda la vida, de forma ubicua y con el empleo creativo de la tecnología con que se cuente (Cabero, 2012; Johnson \& et al, 2016; Sanabria, López \& Leal, 2014; West, 2012).

El rol del profesor ... sigue siendo crucial, si bien ha cambiado su carácter. Actualmente, la misión del educador es cada vez menos la simple transmisión de conocimiento y más la de un mediador entre los estudiantes y la riqueza de información que los bombardea. La Internet, en la medida que se hace más eficiente y accesible, puede ser otro paso en esta dirección, ya que permite la comunicación entre el productor del contenido y el profesor, así como entre los propios profesores, y les brinda una oportunidad de buscar diferentes contenidos (Schwartzman, 2001, p. 40).

En cualquier caso, la formulación de las áreas clave para la formación inicial y permanente del personal docente como un agente esencial que influye en la calidad educativa, con una proyección hacia la sociedad y la cultura del siglo XXI, debe partir de reconocer la integralidad, complejidad y altísima responsabilidad que se acrecienta con los cambios tecnológicos y la necesidad de brindar respuesta a los desafíos sociales de justicia y equidad.

La labor de los profesores es cada vez más compleja y desafiante, deben atender a niños provenientes de sectores diversos de la sociedad, cuyo capital cultural puede diferir de manera importante. Junto con esto, un docente competente no sólo debe conocer su disciplina sino manejar variadas estrategias pedagógicas que permitan a los niños desarrollar habilidades más complejas, actitudes y motivaciones que les permitan participar en la sociedad y convertirse en aprendices autónomos a lo 
largo de la vida; todo esto ha puesto en entredicho las instituciones y prácticas tradicionales de formación inicial y continua de los docentes (OREALC/UNESCO, 2012, p. 73).

\section{Conclusiones}

La calidad de la educación resulta un tema altamente complejo de definir y que aparece condicionado por factores de diversa naturaleza, entre los que cuentan los factores económicos, políticos, culturales, científicos y tecnológicos, así como factores humanos, entre los que destaca el desempeño del personal docente. En todo caso, la calidad de la educación depende de la sociedad y su proyecto de desarrollo, la historia y la cultura; y, también de los niveles de desarrollo de la ciencia y la postura política con que se asuma la educación en cada país. El ideal de calidad se correlaciona, directamente y de modo dialéctico, con el contexto, sus realidades, concreciones, fortalezas, debilidades y metas que deben ser alcanzadas.

El desempeño docente es un factor clave para una educación de calidad. Independientemente de la solvencia económica con que se cuente y el diseño curricular; el desempeño docente, como factor eminentemente humano, es esencial para actuar profesionalmente a la altura de las necesidades de la época y la sociedad en cuestión, y propiciar con la convicción y la responsabilidad necesaria la formación y aprendizajes para toda la vida.

Es urgente asumir la profesión y el desempeño docente como uno de los encargos sociales de mayor responsabilidad y significación a nivel social, razón por la que la escuela no debe ser dejada en manos descuidadas, mal preparadas, desmotivadas o inconscientes de la magnitud del alcance de su labor.

Se sostienen algunos puntos de interés básicos para el análisis correlacional entre el desempeño docente y calidad educativa en la región:

- Las políticas públicas deben resituar con mayor sensibilidad y responsabilidad social la atención a la complejidad del desempeño del personal docente en la contemporaneidad, asumiendo acciones concretas en favor de elevar parejamente su reconocimiento social y el perfil de la profesión, de modo que atraiga a jóvenes con más talentos a los procesos de formación profesional. Entre las acciones deben ser considerados el empleo de incentivos como reconocimiento de resultados, así como el mejoramiento de las condiciones de trabajo, la disponibilidad de tiempo suficiente para investigar, superarse, autosuperarse y preparar material didáctico, entre otros temas necesarios a transformar.

- Estimular el desarrollo de investigaciones y estudios comparados al interior de los países sobre la relación calidad de la educación - desempeño docente en toda la amplia gama de indicadores que pueden ser determinados, medidos y evaluados en correspondencia con el contexto. Es estrictamente necesaria la constitución de instituciones investigativas independientes con tales fines, además de los aportes que pueden brindar las universidades o escuelas normales que tienen como objeto la formación inicial y continua del docentes.

- Propiciar un movimiento de actualización y perfeccionamiento constante de los currículos de manera flexible y contextualizada para propiciar la satisfacción total a las necesidades educativas y preparar a docentes para que enseñen a aprender a lo largo de toda la vida y estimulen la conformación de proyectos de vida para cada niño o niña desde la escuela. En consonancia con la formación inicial de cada docente, debe estar la concepción de la formación continua que conciba una amplia gama de opciones de educación de postgrado, que tengan como base la investigación y la autosuperación. Esta educación de postgrado debe responder, de manera directa, a las necesidades de superación, capacitación, actualización, complementación, profundización y, en sentido general, al desarrollo integral del personal docente. 
- Aplicar de manera contextualizada guías de orientación que propicien de manera sistemática que el personal docente autoevalúe su desempeño y sea este un modo de estimular la elevación de la calidad de la educación.

- Incentivar la innovación y la creatividad en el desempeño docente como un factor dinámico para propiciar ambientes o climas favorables en toda la institución educativa y, con ello, un proceso educativo de mayor complejidad y acción de respuesta responsable a las demandas sociales contemporáneas de carácter regional, nacional y local.

- Adecuar políticas TIC para la educación y el desempeño docente, equilibrando de manera coherente las inversiones y la infraestructura necesaria con el proceso de capacitación, superación y estímulo a docentes, para su empleo creativo y sistemático en su desempeño. El informe Horizon, que se emite cada año, es una guía clara para el diseño de políticas nacionales e institucionales en este sentido.

Estas ideas son sugerencias que soportan distintos niveles de aceptación y de acciones específicas para su concreción, incluso pudieran ser negadas o refutadas desde diferentes lógicas. En cualquier caso, resulta apremiante aceptar el desempeño docente como un factor clave influyente en la calidad de la educación, en tal sentido la reflexión crítica y responsable con la consiguiente toma de acciones en este tema. De este modo, se facilitarían los sustentos teóricos que la educación y la escuela requieren hoy, en un proceso de transformación necesaria y efectiva, como respuesta a la dinámica de los cambios de la sociedad, la economía, la cultura, la ciencia y la tecnología.

\section{REFERENCIAS}

Adams Becker, S., Cummins, M., Davis, A., Freeman, A., Hall Giesinger, C., \& Ananthanarayanan, V.. (2017). The NMC Horizon Report: 2017 Higher Education. España: New Media Consortium (NMC) y EDUCAUSE Learning Initiative (ELI).

Albury, D. (15 de abril de 2013). Tenemos que convertirnos en el cambio educativo. Recuperado de https://unpasomas.fundaciontelefonica.com/2013/04/15/david-albury-tenemos-que-convertirnos-en-elcambio-educativo/

Burchardt, H.-J. (2017). La crisis actual de América Latina: Causas y soluciones. Nueva Sociedad, 267, 114-128.

Cabero, J. (2012). Tendencias para el aprendizaje digital: De los contenidos cerrados al diseño de materiales centrado en las actividades. El Proyecto Dipro 2.0. RED, Revista de Educación a Distancia, 32, 1-27. Obtenido de http:// www.um.es/ead/red/32

Chávez, J. (1995). Filosofía y educación en América Latina. Educación , 14-17.

Delors, J. (s. f.). La educación encierra un tesoro. Informe a la UNESCO de la Comisión Internacional sobre la educación para el siglo XXI. París: Santillana. UNESCO.

Escribano, E. (2017). La educación en América Latina: Desarrollo y perspectivas. Actualidades Investigativas en Educación, 17(2), 1-23. doi:http://dx.doi.org/10.15517/aie.v17i1.28147

Fundación Omar Dengo. (2014). Competencias para el siglo XXI : Guía práctica para promover su aprendizaje y evaluación. San José, Costa Rica: Fundación Omar Dengo.

García, J. (2014). La dinámica de la pobreza en México. Una aproximación multidimensional. Investigación Operacional, 35(2), 141-147.

García, J. F., Caamal, I., \& Prieto, O. (2016). Situación de pobreza y desigualdad en México. Una aproximación teórica. ATENAS, 1(33), 1-17. Obtenido de http://atenas.mes.edu.cu

Gatti, B., \& de Sá Barreto, E. (2009). Professores do Brasil: Impasses e Desafios. Brasilia: UNESCO.

Gatti, B., de Sá Barreto, E., \& André, M. (2011). Políticas docentes no Brasil. Um estado da arte. Brasilia: UNESCOBrasil e Ministério da Educação. 
Gerver, R. (30 de mayo de 2013). La escuela del mañana. Obtenido de https://www.youtube.com/watch? $\mathrm{v}=42$ ReyIvfxZM

Guadarrama, P. (2008). La calidad educativa y la perspectiva ideológica ante la racionalidad instrumental globalizada. Magisterio, 32, 24-28.

Johnson, L., Adams Becker, S., Cummins, M., Estrada, V., Freeman, A., \& Hall, C. (2016). The NMC Horizon Report: 2016 Higher Education Edition. Austin, Texas: The New Media Consortium.

Martínez - Chairez, G. I., Guevara - Araiza, A., \& Valles - Ornelas, M. M. (2016). El desempeño docente y la calidad educativa. Ra Xinhai, 12(6), 123-134. Obtenido de http://www.redalyc.org/articulo.oa?id=46148194007

Martínez, R., Trucco, D., \& Palma, A. (2014). El analfabetismo funcional en América Latina y el Caribe. Santiago de Chile: Naciones Unidas.

Medina, I. L. (2017). El desempeño profesional pedagógico del tutor en la carrera Licenciatura en Educación Pedagogías - Psicología de la Universidad de Matanzas (Tesis doctoral). Matanzas: Universidad de Matanzas.

Morin, E. (2000). Los siete saberes necesarios a la educación del futuro. Caracas: Universidad Central de Venezuela/ IESALC/UNESCO.

OCDE. (2000). La definición y selección de competencias clave. Resumen ejecutivo. Informe DeSeCo. OCDE.

OCDE. (2003). La definición y selección de competencias clave. Resumen ejecutivo . Informe DeSeCo. OCDE.

OCDE. (2010). Habilidades y competencias del siglo XXI para los aprendices del nuevo milenio en los países de la OCDE. España: Instituto de Tecnologías Educativas.

OEI. (2014). Miradas sobre la educación en Iberoamérica. Avances en las metas educativas 2021. Madrid, España: OEI.

Oficina Regional de Educación para América Latina y el Caribe (OREALC/UNESCO). (2013). Situación educativa de América Latina y el Caribe. Santiago de Chile: UNESCO.

OREALC/UNESCO. (2007). Educación de calidad para todos un asunto de derechos humanos. Buenos Aires: Autor.

OREALC/UNESCO. (2012). Situación educativa de América Latina y el Caribe. Santiago: OREALC/UNESCO.

OREALC/UNESCO. (2013). Antecedentes y criterios para la elaboración de políticas docentes en América Latina y el Caribe. Santiago de Chile: UNESCO.

OREALC/UNESCO. (2016). Informe de resultados TERCE. Tercer estudio regional comparativo y explicativo. Logros de aprendizaje. Santiago de Chile: UNESCO.

P21 Framewok for 21st Century Learning. (2015). 21st Century Student Outcomes. USA: Consorcio de Habilidades Indispensables para el siglo XXl.

Parra, I. V. (2000). Desarrollo de la competencia didáctica del profesional de la educación en formación inicial (Tesis doctoral). Universidad de Ciencias Pedagógicas “Enrique José Varona”, La Habana. : Instituto Superior Pedagógico "Enrique José Varona”.

Patti, J., Brackett, M., Ferrándiz, C., \& Ferrando, M. (2011). ¿Por qué y cómo mejorar la inteligencia emocional de los alumnos superdotados? Revista Electrónica Interuniversitaria de Formación del Profesorado, 14(3), 145-156. Obtenido de http://www.aufop.com

Pérez, F. (2003). Pobreza y exclusión: Principales actores de la problemática educativa latinoamericana y caribeña. Cuadernos de Nuestra América, 16(31), 95-125.

Pérez, F. A.(2009). Un modelo para el desempeño profesional del docente de preuniversitario. La Habana: ICCP.

Pérez, R. J. (2008). Conocimientos y habilidades en las competencias laborales . La Habana: EHTH.FORMATUR.

Plá, S. (2014). Debates contemporáneos sobre la inequidad y la exclusión educativa en América Latina. Sinéctica, 44, 1-19. Obtenido de www.sinectica.iteso.mx

Rivas, A. (2015). América Latina después de PISA. Lecciones aprendidas en siete países (2000-2015). Buenos Aires: Fundación CIPPEC.

Robinson, K. (2011). El sistema educativo del siglo XIX en pleno siglo XXI. (E. Punset, Entrevistador) Recuperado de https://www.youtube.com/watch?v=X2nWP9bztWM 
Román, M., \& Murillo, F. (2008). La evaluación del desempeño docente: Objeto de disputa y fuente de oportunidades en el campo educativo. Revista Iberoamericana de Evaluación Educativa, 1(2), 1-6. Obtenido de http:// www.rinace.net/riee/numeros/vol1-num2/editorial.pdf

Sanabria, L., López, O., \& Leal, L. (2014). Desarrollo de competencias metacognitivas e investigativas en docentes en formación mediante la incorporación de tecnologías digitales: Aportes a la excelencia docente. Revista Colombiana de Educación, 67, 147-170.

Savater, F. (1997). El valor de educar. Barcelona: Ariel S. A.

Schwartzman, S. (2001). El futuro de la educación en América Latina y el Caribe. Santiago de Chile: UNESCO.

Siemens, G. (18 de octubre de 2012). Blogs de cultura digital e innovación. Obtenido de https:// unpasomas.fundaciontelefonica.com/2012/10/18/conectivismo-george-siemens-y-el-aprendizaje-en-red/

SITEAL. (2013). El analfabetismo en América Latina. Buenos Aires: OEI, UNESCO, Instituto Internacional de Planeamiento para la Educación.

SITEAL. (2014). Políticas TIC en los sistemas educativos en América Latina. Buenos Aires: OEI, UNESCO, Instituto Internacional de Planeación Educativa.

Sunkel, G., Trucco, D., \& Espejo, A. (2013). La integración de las tecnologías digitales en las escuelas de América Latina y el Caribe. Santiago de Chile: Naciones Unidas y CEPAL.

Tedesco, J. C. (Septiembre de 2010). Educación para una sociedad más justa. Buenos Aires, Argentina. Recuperado de https://www.youtube.com/watch?v=WDhLvL5N4HU

Tobón, S., González, L., Nambo, J., \& Vázquez, J. (2015). La socioformación: Un estudio conceptual. Paradigma, $36(1), 7-29$.

Torres, C. A. (2001). Grandezas y miserias de la educación latinoamericana del siglo veinte. En C. L. CLACSO, Paulo Freire y la agenda de la educación latinoamericana en el siglo XXI (pp. 23-52). Buenos Aires: CLACSO, Consejo Latinoamericano de Ciencias Sociales. Obtenido de http://bibliotecavirtual.clacso.org.ar/clacso/ gt/20101010031230/2torres.pdf

UNESCO. (2013). Uso de TIC en educación en América Latina y el Caribe. Análisis regional de la integración de las TIC en la educación y de la aptitud digital (e-readiness). Montreal: Instituto de Estadística de la UNESCO. Obtenido de http://www.uis.unesco.org

Vaillant, D. (2007). Mejorando la formación y el desarrollo profesional docente en Latinoamérica. Rev. Pensamiento Educativo, 41(2), 1-16.

Van de Velde, H. (2014). Construyendo escenarios educativos basados en cooperación genuina. Estelí, Nicaragua: ABACOenRed.

Van de Velde, H. (2016). Apuntes sobre calidad educativa... ¿Cuestión de producto y/o de proceso? Estelí, Nicaragua: ABACOenRed. Obtenido de www.abacoenred.com

West, M. (2012). Aprendizaje movil para docentes. Temas globales. París: UNESCO.

\section{BY-NC-ND}

